\title{
Chest CT in the emergency department for suspected COVID-19 pneumonia
}

\author{
Anna Palmisano ${ }^{1,2}$. Giulia Maria Scotti ${ }^{3}$. Davide Ippolito ${ }^{4,5} \cdot$ Marco J. Morelli $^{3}$. Davide Vignale ${ }^{1,2}$. \\ Davide Gandola $^{4,5}$. Sandro Sironi ${ }^{5,6} \cdot$ Francesco De Cobelli $^{1,2} \cdot$ Luca Ferrante $^{7} \cdot$ Marzia Spessot $^{7} \cdot$ Giovanni Tonon $^{3}$. \\ Carlo Tacchetti ${ }^{1,2} \cdot$ Antonio Esposito $^{1,2}$ (1)
}

Received: 4 June 2020 / Accepted: 20 October 2020 / Published online: 9 November 2020

(c) Italian Society of Medical Radiology 2020

\begin{abstract}
Purpose In overwhelmed emergency departments (EDs) facing COVID-19 outbreak, a swift diagnosis is imperative. CT role was widely debated for its limited specificity. Here we report the diagnostic role of CT in two EDs in Lombardy, epicenter of Italian outbreak.

Material and methods Admitting chest CT from 142 consecutive patients with suspected COVID-19 were retrospectively analyzed. CT scans were classified in "highly likely," "likely," and "unlikely" COVID-19 pneumonia according to the presence of typical, indeterminate, and atypical findings, or "negative" in the absence of findings, or "alternative diagnosis" when a different diagnosis was found. Nasopharyngeal swab results, turnaround time, and time to positive results were collected. CT diagnostic performances were assessed considering RT-PCR as reference standard.

Results Most of cases (96/142, 68\%) were classified as "highly likely" COVID-19 pneumonia. Ten (7\%) and seven (5\%) patients were classified as "likely" and "unlikely" COVID-19 pneumonia, respectively. In 21 (15\%) patients a differential diagnosis was provided, including typical pneumonia, pulmonary edema, neoplasia, and pulmonary embolism. CT was negative in 8/142 (6\%) patients. Mean turnaround time for the first COVID-19 RT-PCR was 30 $\pm 13 \mathrm{~h}$. CT diagnostic accuracy in respect of the first test swab was 79\% and increased to $91.5 \%$ after repeated swabs and/or BAL, for 18 false-negative first swab. CT performance was good with $76 \%$ specificity, $99 \%$ sensitivity, $90 \%$ positive predictive value and $97 \%$ negative predictive value.

Conclusion Chest CT was useful to streamline patients' triage while waiting for RT-PCR in the ED, supporting the clinical suspicion of COVID-19 or providing alternative diagnosis.
\end{abstract}

Keywords COVID-19 $\cdot$ Diagnosis $\cdot$ Computed tomography $\cdot$ Triage $\cdot$ Emergency

Antonio Esposito

esposito.antonio@hsr.it

1 Experimental Imaging Centre, Radiology Unit, IRCCS San Raffaele Scientific Institute, Milan, Italy

2 School of Medicine, Vita-Salute San Raffaele University, via Olgettina 58, 20132 Milan, Italy

3 Center for Omics Sciences, IRCCS San Raffaele Scientific Institute, Milan, Italy

4 Diagnostic Radiology, San Gerardo Hospital, School of Medicine and Surgery, Monza, Italy

5 University of Milano-Bicocca, School of Medicine and Surgery, Monza, Italy

6 Department of Radiology, ASST Papa Giovanni XXIII, Bergamo, Italy

7 Emergency Medicine, Emergency Department, IRCCS San Raffaele Scientific Institute, Milan, Italy

\section{Chest CT in the emergency department for suspected COVID-19 pneumonia}

SARS-CoV-2 spread worldwide causing coronavirus disease 2019 (COVID-19) which may overlap seasonal flu and other respiratory diseases. COVID-19 lung involvement progresses toward a severe pneumonia in $15-20 \%$ of cases, requiring critical care in $5-10 \%$ of them [1]. Thus, expeditious and reliable diagnosis is imperative.

The cornerstone of COVID-19 diagnosis is RT-PCR from oral-nasopharyngeal swab. RT-PCR has near-perfect specificity, but limited sensitivity (30-70\%) varying according to viral burden, symptoms severity, and time from symptoms onset [2]. Moreover, the turnaround time for results may be long in overwhelmed laboratories, as during epidemic 
growth [3]. Chest imaging showed good performance in COVID-19 pneumonia diagnosis [4-6], with chest X-ray representing the first line due to wide availability and portability [6], and CT outperforming RT-PCR sensitivity even in the early stages of the disease [5].

Despite the high sensitivity, the usage of CT to triage symptomatic patients with suspected COVID-19 pneumonia was questioned for a matter of specificity, being CT findings potentially overlapping to other viral pneumonia [7]. The aim of the study was to investigate the value of chest CT in triaging patients with suspected COVID-19 pneumonia in two emergency departments (EDs) in Lombardy, Italy.

We retrospectively analyzed 142 chest CT scans performed from March 8 to 30, 2020, at two tertiary-care University Hospitals in patients referring to the EDs for suspected COVID-19 pneumonia, with inconclusive or possible false-negative X-ray results [8]. Chest CT was performed on a dedicated scanner with a separated access applying standardized protocols for infection control.

According to RSNA Expert Consensus [9], chest CT scans were classified into: (i) "highly likely COVID-19" in the presence of typical findings, (ii) "likely COVID-19" in the presence of indeterminate findings, (iii) "unlikely COVID-19" in the presence of atypical findings for
COVID-19, (iv) "alternative diagnosis" in case of findings indicative for a different causes of symptoms, and (v) "negative" (Fig. 1).

Nasopharyngeal swabs results were collected, including turnaround time, number of swabs performed, and timeto-positive results.

CT sensitivity, specificity, positive predictive value (PPV), negative predictive value (NPV), and accuracy were measured, considering results from repeated swabs or bronchoalveolar lavage (BAL) as reference standard.

Statistical analyses were performed using SPSS v.25.0.

All 142 patients $(M=101$; median age 61 y.o. [interquartile range (IQR) 50-73]) suffered from fever ( $\geq 37.5^{\circ} \mathrm{C}$ from a median of 7 days [IQR 3-10]), associated most frequently with cough $(75 / 142,53 \%)$, and dyspnea $(45 / 142,32 \%)$. Acute chest pain and gastrointestinal symptoms were rare (8/142, 6\%, in both cases). At arrival, median oxygen saturation ( $\mathrm{SatO} 2)$ was 94\% [89-96\%].

$\mathrm{CT}$ and swab results are summarized in Fig. 2.

Chest CT was acquired without contrast, except in 8 patients to exclude vascular events.

Chest CT showed typical findings for COVID-19 pneumonia in most of cases $(96 / 142,68 \%)$, while it was
Fig. 1 Exemplifying CT findings for each class of COVID19 pneumonia probability based on the presence of typical, indeterminate and atypical finding, or eventual absence of signs of viral pneumonia and alternative $\mathrm{CT}$ diagnosis. A is reported unenhanced thin-section axial CT image showing typical peripheral ground-glass opacity with superimposed interlobular septal thickening and intralobular lines visibility ("crazypaving" pattern), involving both lungs. Unilateral involvement, considered one of the indeterminate features, is reported in B. Atypical findings (C) include bronchiolar wall thickening and tree-in-bud opacities with centrilobular nodules (arrows in C). Finally, alternative diagnosis (D) was reported in the case of chest CT findings not typical for interstitial pneumonia and with clear pulmonary or extrapulmonary findings explaining symptoms and laboratory test alteration (e.g., pulmonary neoplasia, red arrow in D, associated with bilateral pleural and pericardial effusion)

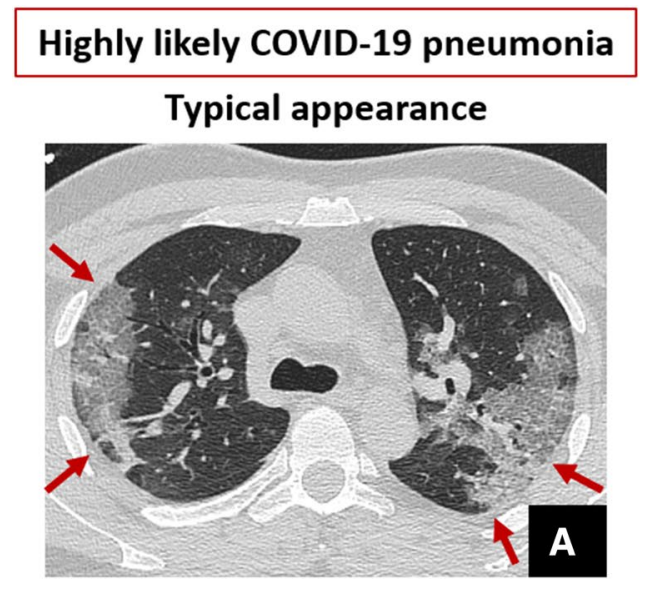

\section{Probable COVID-19 pneumonia}

Indeterminate appearance

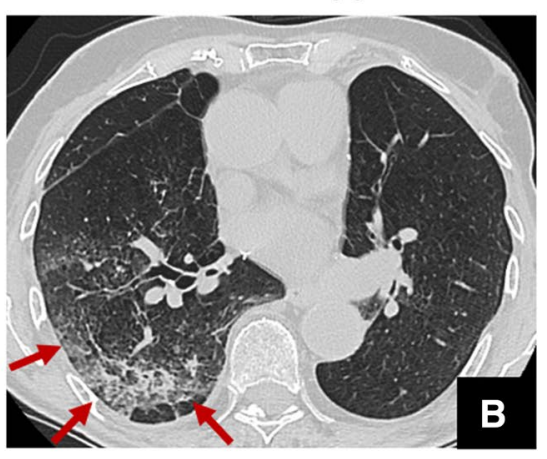

Unlikely COVID-19 pneumonia Atypical appearance
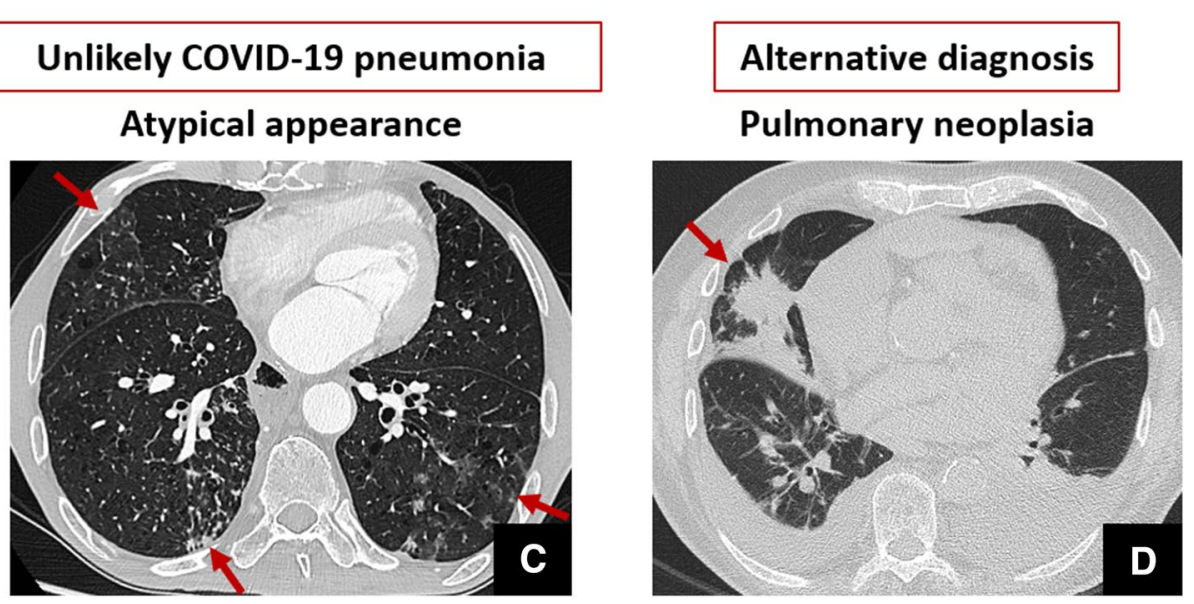


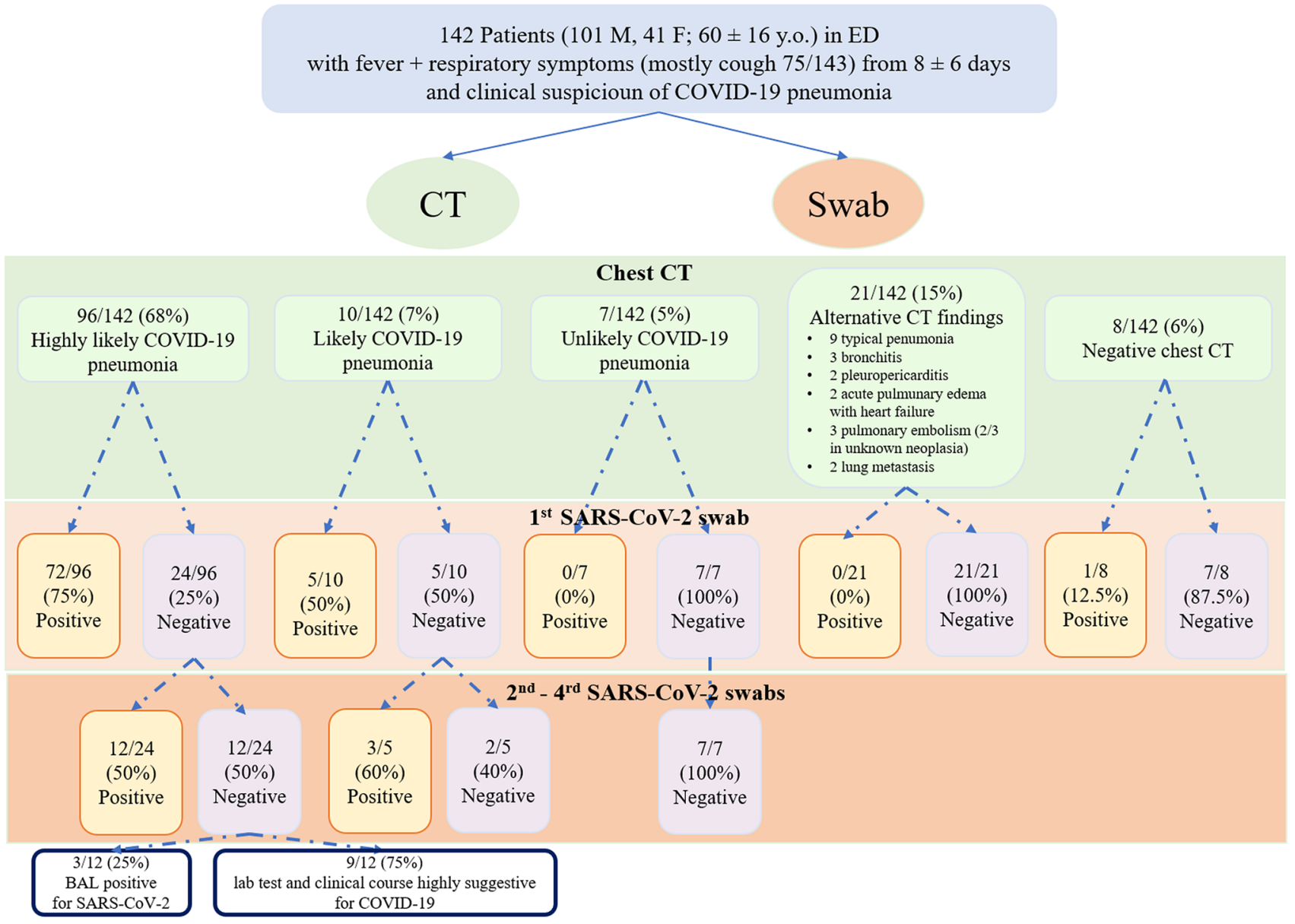

Fig. $2 \mathrm{CT}$ and swabs results in triaging patients with suspected COVID-19 pneumonia. Flowchart of CT findings and nasopharyngeal swab results in 142 febrile patients attending the EDs of two hospital in Lombardy with clinical suspicious of SARS-CoV2 infection. The agreement between CT and RT-PCR increased after first swab, for a

classified as "likely COVID-19 pneumonia" in 10/142 (7\%), "unlikely" in 7/142 (5\%), and "negative" in 8/142 $(6 \%)$.

In $15 \%$ of patients (21/142) CT showed alternative diagnosis including lobar pneumonia, pulmonary edema, and neoplasia.

Turnaround time for first RT-PCR result was $30 \pm 13 \mathrm{~h}$ (range $2-48 \mathrm{~h}$ ). A median of 2 swabs was performed per patient.

Swab was negative for SARS-CoV2 infection in all CT scan classified as "unlikely" and "alternative diagnosis," while was positive in a single case of "negative" CT.

The first swab was positive in 77/106 (73\%) and negative in $29 / 106(27 \%)$ patients classified as "highly likely" and "likely" at CT. In 18/29 (62\%) patients, negative swabs turned to positive after repetition or BAL (mean time for positivization: $5 \pm 4$ days) (Fig. 3 ). In the remaining 9 patients, laboratory test and clinical course were highly total of 18 patients initially false negative at RT-PCR on nasopharyngeal swab. Interestingly, CT in addition to the high sensitivity had the advantage to provide differential diagnosis useful for patients' clinical management

suggestive for COVID-19. In one case classified as "likely COVID-19 pneumonia" H1N1 virus was isolated.

CT diagnostic accuracy was 79\% in respect of first swab and raised to $91.5 \%$ after repeated swabs and/or BAL.

Chest CT performance in triaging symptomatic patients suspected for COVID-19 was good with $76 \%$ specificity, 99\% sensitivity, 90\% PPV, and 97\% NPV.

Our data showed that during the rise of COVID-19 epidemic the integration of chest $\mathrm{CT}$ in triaging patients has high PPV and NPV in line with previous results [10, 11], with higher sensitivity than swab [5], which often needed to be repeated and is affected by long turnaround time.

One false negative was observed in a patient with CT performed within 2 days of symptoms onset, probably related to the known lower prevalence of parenchymal involvement in the earliest stages of disease $[12,13]$. In our setting, CT specificity and PPV were slightly higher than in most previous reports $[5,10]$, probably in relation to the better clinical 


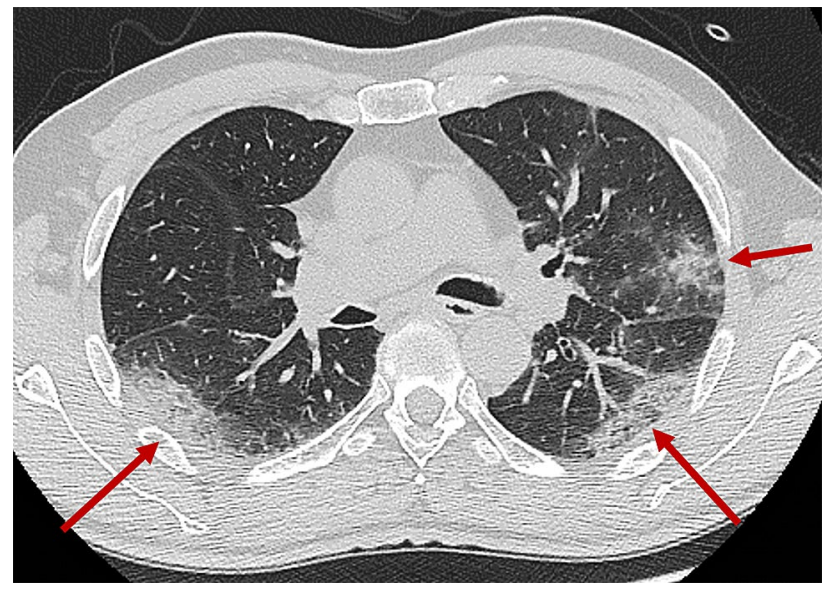

Fig. 3 Chest CT typical COVID-19 pneumonia in a patient with initially negative swab. A 61-year-old man suffering from fever $\left(39^{\circ} \mathrm{C}\right)$, cough, and dyspnea from 7 days, presented to the emergency department of San Raffaele Hospital in Milan. Clinical evaluation and laboratory tests resulted highly suspicious for SARS-CoV2-related pneumonia. Nasopharyngeal swab and chest CT were immediately performed. CT showed peripheral opacity with crazy-paving pattern and consolidation (red arrows) mainly involving the upper left lobe and the lower lobes, mainly with posterior distribution. CT findings resulted highly suggestive for SARS-CoV2 pneumonia, but results from the first swab (available only $24 \mathrm{~h}$ later) resulted negative. In consideration of high clinical and CT suspicion, another swab was collected after 3 days, and it finally resulted positive

selection of patients and high pretest probability of disease in epidemic territories $[8,11]$.

Our findings highlight the unique capability of $\mathrm{CT}$ to provide alternative diagnoses in a substantial number of cases, which cannot be addressed with chest X-ray. In fact, although COVID-19 pneumonia was the prevalent causes of respiratory dysfunction during the outbreak, it was not the unique.

In agreement with the recent Fleischner Society expert consensus document [14], our data support the integration of $\mathrm{CT}$ in triaging suspected patients because it may streamline decision making in overwhelmed EDs, where oral-nasopharyngeal swab may not yet provide trustworthy and fast results, and avoiding exposure in non-COVID-19 patients.

Importantly, one case of $\mathrm{H} 1 \mathrm{~N} 1$ pneumonia was reported, for the known possibility of overlapping CT findings, highlighting the necessity to provide a microbiological confirmation also in patients with highly suggestive CT findings [8].

Our findings are applicable in a scenario of high prevalence of disease (around 40\%), as occurred during the ascending phase of epidemic curve [11]; therefore, they cannot be generalized to low prevalence situation where could be expected a poor PPV [11].

The application of chest CT in triage suspected COVID19 patients is related to the availability of a dedicated scanner, because long downtime (30-60 min after imaging a suspected patient) for room decontamination and passive air exchange may significantly interfere with routine clinical activity.

In conclusion, in the specific context of the overwhelmed EDs facing COVID-19 outbreak, chest CT may fasten triaging, while waiting for RT-PCR results, by supporting the clinical suspicion of COVID-19 or providing an alternative diagnosis with overlapped clinical presentation.

Acknowledgments This work was supported by the Italian Ministry of Health with $5 \times 1000$ funds and by OSR Program COVID-19.

\section{Compliance with Ethical Standards}

Conflict of interest Authors have no conflict of interest to disclose.

Ethical standards Our research study includes human participants. The study protocol complies with the Declaration of Helsinki and was approved by the local hospital ethics committee (protocol no. 34/ int/2020). Patients able to provide a signed informed consent were consented prior to data collection. The ethics committee waived the requirement for obtaining written informed consent for patients who had died or were unreachable after discharge.

\section{References}

1. Wang D, Hu B, Hu C, Zhu F, Liu X, Zhang J, Wang B, Xiang H, Cheng Z, Xiong Y, Zhao Y, Li Y, Wang X, Peng Z (2020) Clinical characteristics of 138 hospitalized patients with 2019 novel coronavirus-infected pneumonia in Wuhan, China. JAMA 323(11):1061-1069. https://doi.org/10.1001/jama.2020.1585

2. Wang W, Xu Y, Gao R, Lu R, Han K, Wu G, Tan W (2020) Detection of SARS-CoV-2 in different types of clinical specimens. JAMA. https://doi.org/10.1001/jama.2020.3786

3. Reusken C, Broberg EK, Haagmans B, Meijer A, Corman VM, Papa A, Charrel R, Drosten C, Koopmans M, Leitmeyer K, On Behalf Of E-L, Erli N (2020) Laboratory readiness and response for novel coronavirus (2019-nCoV) in expert laboratories in 30 EU/EEA countries, January 2020. Euro Surveill 25(6). https://doi. org/10.2807/1560-7917.ES.2020.25.6.2000082

4. Borghesi A, Zigliani A, Masciullo R, Golemi S, Maculotti P, Farina D, Maroldi R (2020) Radiographic severity index in COVID-19 pneumonia: relationship to age and sex in 783 Italian patients. Radiol Med 125(5):461-464. https://doi.org/10.1007/ s11547-020-01202-1

5. Ai T, Yang Z, Hou H, Zhan C, Chen C, Lv W, Tao Q, Sun Z, Xia $\mathrm{L}$ (2020) Correlation of chest CT and RT-PCR testing in coronavirus disease 2019 (COVID-19) in China: a report of 1014 cases. Radiology:200642. https://doi.org/10.1148/radiol.2020200642

6. Cozzi D, Albanesi M, Cavigli E, Moroni C, Bindi A, Luvara S, Lucarini S, Busoni S, Mazzoni LN, Miele V (2020) Chest X-ray in new coronavirus disease 2019 (COVID-19) infection: findings and correlation with clinical outcome. Radiol Med 125(8):730-737. https://doi.org/10.1007/s11547-020-01232-9

7. ACR Recommendations for the use of Chest Radiography and Computed Tomography (CT) for Suspected COVID-19 Infection. Accessed 14 April 2020

8. Neri E, Miele V, Coppola F, Grassi R (2020) Use of CT and artificial intelligence in suspected or COVID-19 positive patients: statement of the Italian Society of Medical and Interventional 
Radiology. Radiol Med 125(5):505-508. https://doi.org/10.1007/ s11547-020-01197-9

9. Simpson S, Kay FU, Abbara S, Bhalla S, Chung JH, Chung M, Henry TS, Kanne JP, Kligerman S, Ko JP, Litt H (2020) Radiological society of North America expert consensus statement on reporting chest CT findings related to COVID-19. Endorsed by the Society of Thoracic Radiology, the American College of Radiology, and RSNA. Radiol Cardiothoracic Imaging 2(2):e200152. https://doi.org/10.1148/ryct.2020200152

10. Kim H, Hong H, Yoon SH (2020) Diagnostic performance of CT and reverse transcriptase-polymerase chain reaction for coronavirus disease 2019: a meta-analysis. Radiology:201343. https://doi. org/10.1148/radiol.2020201343

11. Esposito A, Palmisano A, Scotti GM, Morelli MJ, Vignale D, De Cobelli F, Tonon G, Tacchetti C (2020) Why is chest CT important for early diagnosis of COVID-19? Prevalence matters. medRxiv

12. Pan F, Ye T, Sun P, Gui S, Liang B, Li L, Zheng D, Wang J, Hesketh RL, Yang L, Zheng C (2020) Time course of lung changes on chest CT during recovery from 2019 novel coronavirus (COVID-19) pneumonia. Radiology:200370. https://doi. org/10.1148/radiol.2020200370
13. Wang Y, Dong C, Hu Y, Li C, Ren Q, Zhang X, Shi H, Zhou M (2020) Temporal changes of CT findings in 90 patients with COVID-19 pneumonia: a longitudinal study. Radiology:200843. https://doi.org/10.1148/radiol.2020200843

14. Rubin GD, Ryerson CJ, Haramati LB, Sverzellati N, Kanne JP, Raoof S, Schluger NW, Volpi A, Yim JJ, Martin IBK, Anderson DJ, Kong C, Altes T, Bush A, Desai SR, Goldin J, Goo JM, Humbert M, Inoue Y, Kauczor HU, Luo F, Mazzone PJ, Prokop M, Remy-Jardin M, Richeldi L, Schaefer-Prokop CM, Tomiyama N, Wells AU, Leung AN (2020) The role of chest imaging in patient management during the COVID-19 pandemic: a multinational consensus statement from the fleischner society. Chest. https:// doi.org/10.1016/j.chest.2020.04.003

Publisher's Note Springer Nature remains neutral with regard to jurisdictional claims in published maps and institutional affiliations. 